\title{
Online Learning in Engineering Courses Using Wireless Sensor and Actuator Networks
}

\author{
http://dx.doi.org/10.3991/ijep.v3iS1.2457 \\ A. Cardoso ${ }^{1}$ and P. Gil ${ }^{2}$ \\ ${ }^{1}$ University of Coimbra, Coimbra, Portugal \\ 2 Universidade Nova de Lisboa, Lisboa, Portugal
}

\begin{abstract}
The Information and Communication Technologies have been increasingly playing an essential role to support teaching and learning, especially in engineering courses, by taking advantage of online resources and online experiments. In particular, Wireless Sensor and Actuator Networks are simultaneously a subject of interest for several engineering courses and a way to enable remote access to laboratory experimental setups. The Remote and Virtual Laboratory of the Department of Informatics Engineering of the University of Coimbra (Portugal) is part of the flock.uc.pt web-based platform under development to allow users to perform remote and virtual experiments in several engineering domains. This paper aims to describe its potential use mainly in engineering courses and lifelong learning programs. The main characteristics of some remote experiments and the potential of Wireless Sensor and Actuator Networks to interact with laboratory systems are described.
\end{abstract}

Index Terms-online learning, online experimentation, remote and virtual lab, engineering courses, wireless communications, wireless sensor and actuator networks.

\section{INTRODUCTION}

Higher education institutions must start delivering on its promise of providing learning experiences that engage and address the needs of society in the twenty-first century [1]. As Swail [2] states, the "rules are changing and there is increased pressure on institutions of higher education to evolve, adapt, or desist”. The transformation of teaching and learning on higher education is inevitable with the use of web-based communications technology [3]. The field of online learning, in general, should incorporate the potential of technology and new pedagogical methodologies to address the challenges associated with providing a high quality learning experience in different educational contexts, and using diverse technological supports and interfaces.

The widespread availability of broadband Internet connection has pushed educators to use innovative ways to support learning [4], not only in terms of contents, but also in what the available tools and platforms is concerned. A major outcome from this effort is the online laboratory paradigm. Nowadays, several higher education institutions offer, in addition to their traditional laboratories and presential interaction with setups, online labs in different scientific fields. Similar to traditional laboratories, this type of labs provides students with particular engineering experiences and allows them to explore systems and their real behaviors.
In fact, online labs can be extremely useful for carrying out remote or virtual experiments and can add significant value to online learning programs. Other possibilities for using online labs concern the situations for which teachers want to interact with laboratory systems during classes to demonstrate some concepts or explain how to perform an experiment. Furthermore, they enable students to acquire introductory hands-on experience and to get familiar with real-life equipment prior to laboratory practice. In this way, efforts could be focused more in the experiment itself rather than in the familiarization with the lab equipment [5].

In the last few decades there have been considerable and consistent efforts in developing remote and virtual labs (RVL) as web-based education tools and platforms for students and technicians in lifelong learning programs, where the inherent flexibility and adaptability of such systems are greatly valued [6]. Furthermore, the incorporation of Wireless Sensor and Actuator Networks (WSAN) will provide an extra degree of flexibility to the underlying paradigm, in particular by allowing the extension of the physical local area network, and by enabling to perform experiments over a local wireless network based on wireless sensors and actuators.

Nowadays, WSAN constitute a flexible and accurate interface to remotely acquire/send data from/to a laboratory system. They can be found in a large range of application areas, such as in process industry, healthcare, environment monitoring, communications and education [7].

Usually, WSAN are networks involving low cost devices comprising a processing unit with limited computational power and limited memory that can sense, interact with external sensors and actuators and communicate using wireless communication protocols (Fig. 1). They have their own energy resources and are able to be deployed almost in any environment, supporting different topologies, which enhance the dynamic behavior of the WSAN. Each device is called a mote or sensor node and each network has one special node called sink that interfaces with, for example, a personal computer through a USB connector. Therefore, WSAN allow the interaction with lab equipment assuming the availability of a local network (connecting a class room and the lab room) or an Internet connection.

The Remote and Virtual Laboratory at the Department of Informatics Engineering of the University of Coimbra (Portugal) is a hybrid platform that aims to allow users to carry out remote and/or virtual experiments [8]. 


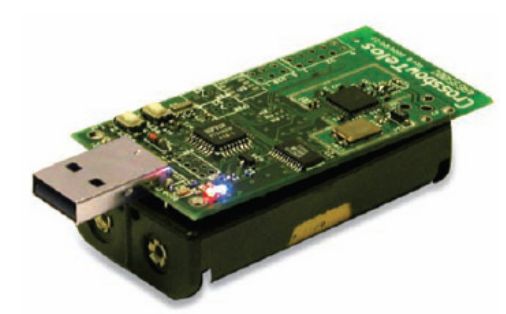

Figure 1. Example of a wireless sensor and actuator node.

This RVL is part of the flock.uc.pt web platform in development to offer online courses, mainly supported on online experimentation, for secondary education, engineering and lifelong learning courses.

Next sections outline the main characteristics of the FLOCK web platform and present some experiments using WSAN that can be considered for teaching or in online learning activities, essentially in engineering courses.

\section{THE FLOCK WEB PlATFORM}

FLOCK (Flexible Learning \& Online Collaborative Knowledge) is a web platform (http://flock.uc.pt) to provide online modules or courses, especially using online experimentation, for different users, from secondary to higher education, and also to lifelong training, and pursuing to offer a collaborative framework where tutors and students/trainees can communicate and interact remotely through the internet, in a b- or e-learning context.

\section{A. The Platform Structure}

The flock.uc.pt platform (Fig. 2) uses Moodle as Learning Management System (LMS) to take advantage of its main dynamic learning environment features to design a course or module. The templates for some of the educational modules were developed by applying design techniques to improve the analytical capabilities of perception and cognition, trying to enhance and promote the learning process [9].

The requirements for the platform involve the integration of different type of courses and educational modules in Portuguese and English, the online experimentation using remote and virtual experiments, the possibility of interaction with haptic devices and the support for an intelligent tutorial system with mechanisms of identification, monitoring and supervision of the users' behavior and knowledge enhancement.

For virtual experimentation, the development of a tool based on 2D/3D game design environments is in progress. The objectives are to provide the user with different forms of interaction, attracting and facilitating the learning process, and to give the user the ability to interact with virtual objects in an immersive environment and, possibly, to receive feedback through physical responses of haptic devices, based on certain attributes of the corresponding real objects.

In the web-based virtual laboratory, students or trainees can perform experiments on setups expressed in the form of models of systems, being the virtual system's time response emulated by simulation. On the other hand, using the remote laboratories, users can have access to real pilot plants, connected via data acquisition boards or wireless sensor networks to a server computer.

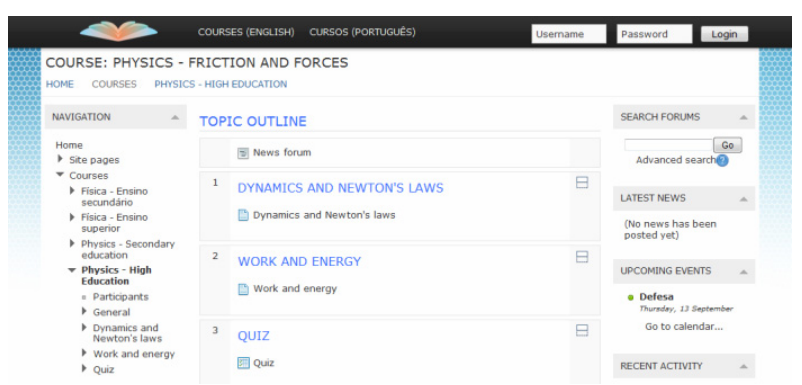

Figure 2. Example of a course's interface at flock.uc.pt web platform.

\section{B. The Remote Lab}

The remote lab is a hybrid laboratory with interactive computer applications that allows carrying out remote experiments using real laboratory systems available at the Laboratory of Industrial Informatics and Systems of the Department of Informatics Engineering of the University of Coimbra. The experiments include monitoring systems observing physics variables, systems identification, digital control of dynamic systems, networked control systems and distributed control systems, considering remote controllers in a shared communication network.

The remote monitoring and control of different systems can be implemented using a WSAN in a distributed framework, where sensors and actuators are spatially distributed and connected through nodes of the wireless network to a gateway that provides the data to the main platform or to a local computer.

\section{The WSAN Framework}

The remote laboratory is based on a client-server architecture where the connection with the pilot plants can be implemented through wired Data Acquisition boards (DAQ) or a WSAN (Fig. 3), and includes four main applications. The server application is running on a server computer at the laboratory and the client application is installed on a remote computer. Additionally, the media server application runs on another computer and the process application is running on each computer connected to a real process.

When the connections between the sensors and the server computer use a WSAN (links 3 and 4 in Fig. 4), the underlying physical variables are collected locally by specific transducers and sent to the sink node using the available network of wireless sensor nodes. The output from the transmitters, namely temperature, level or flow rate transmitters, must be converted into a voltage signal in a range accordingly to the sensor node characteristics (for example, 0 to $2.5 \mathrm{~V}$ ), before providing it to the sensor nodes' ADC channels. For actuation purposes, the data is sent from the server to a local node through the sink node (links 1 and 2 in Fig. 4) and the system input variables are controlled connecting the voltage signals from the node's DAC channels to the transmitters associated to the actuators.

\section{The WSAN Simulator}

To design the WSAN and predict its behavior and performance, a simulation platform (Fig. 5) based on the TrueTime simulator [10] was developed. Its features provide a very flexible simulation environment with application in different scientific areas, such as, WSAN communication protocols or monitoring and control over wireless communication networks [11]. 


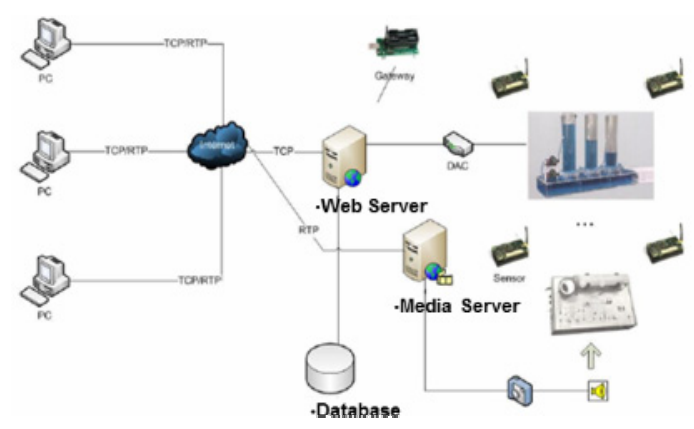

Figure 3. Remote Laboratory Architecture.

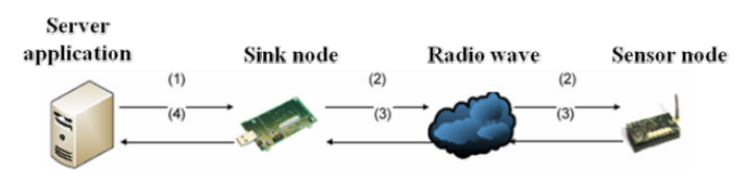

Figure 4. Wireless connection between a sensor node and a server computer.

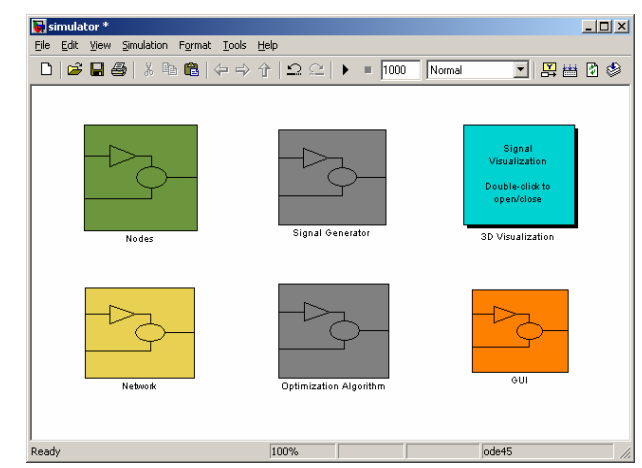

Figure 5. Main WSAN simulation platform interface.

The most relevant features of this simulator engine include graphical representation of communication components, wireless communication and battery-driven operation. Special attention was paid to 3D network visualization, interactivity and extendibility of this platform. Another useful feature is its inherent ability to interact with real motes in a real WSAN.

\section{EXPERIMENTS}

Some remote and virtual experiments will be available through the flock.uc.pt web platform. The next four experiments are examples of remote labs that can be implemented using a WSAN to collect/send data from/to the didactic systems.

\section{A. Experiment 1: Monitoring a SISO nonlinear process}

The purpose of this experiment is to monitor the behavior of a Single-Input-Single-Output (SISO) nonlinear system, using a wireless sensor network (WSN) to observe remotely the system output. The temperature control process trainer (model PT326 from Feedback, Fig. 6 ) is an example of a system that could be incorporated in this remote experiment.

The PT326 system comprises a heating element controlled by a thyristor circuit that feeds heat into the air stream circulated by a fan along a tube. A thermistor detector, which may be placed at one of three points along the tube length, senses the temperature. The volume of air flow is established manually varying the input area by means of a damper.

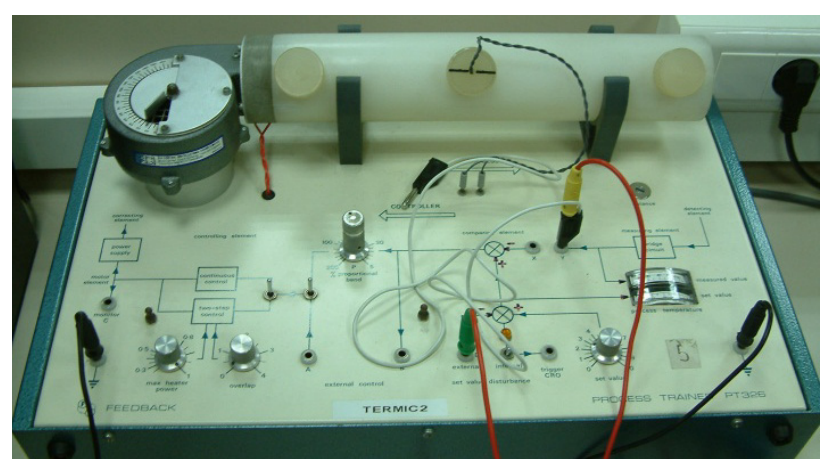

Figure 6. The temperature control process trainer (PT326) used in Experiment 1.

Remote monitoring of temperature using a WSN is an example of a remote experiment that can be integrated in engineering courses for different subjects. In this framework, additional sensors spatially distributed to read several temperatures could be connected through nodes of the wireless network to a gateway that provides the data to the platform.

\section{B. Experiment 2: Control of a SISO nonlinear process}

The objective of this experiment is to control a SISO nonlinear system, implementing different types of controllers and considering a WSN to acquire the system input and output.

The two-tank system (Armfield - PCT9) is one of the systems considered in this experiment. The system (Fig. 7) comprises a motorized valve to control the flow rate of water pumped from a sump tank to a dual compartment tank at the top of the plant, where a sensor measures the liquid level. Several outputs with different flow rates can be chosen. In this case, the system is locally controlled and the WSN is used to monitor its behavior and the controller performance, observing the data collected from the sensors.

\section{Experiment 3: Remote Control of a MIMO system}

This experiment aims to introduce a non-dedicated communication network between the controller and a Multi-input-Multi-Output (MIMO) nonlinear system to build a networked environment. Using the Internet to remotely control a real system, it is possible to analyze the robustness of the overall system to delays and faults in the communication network.

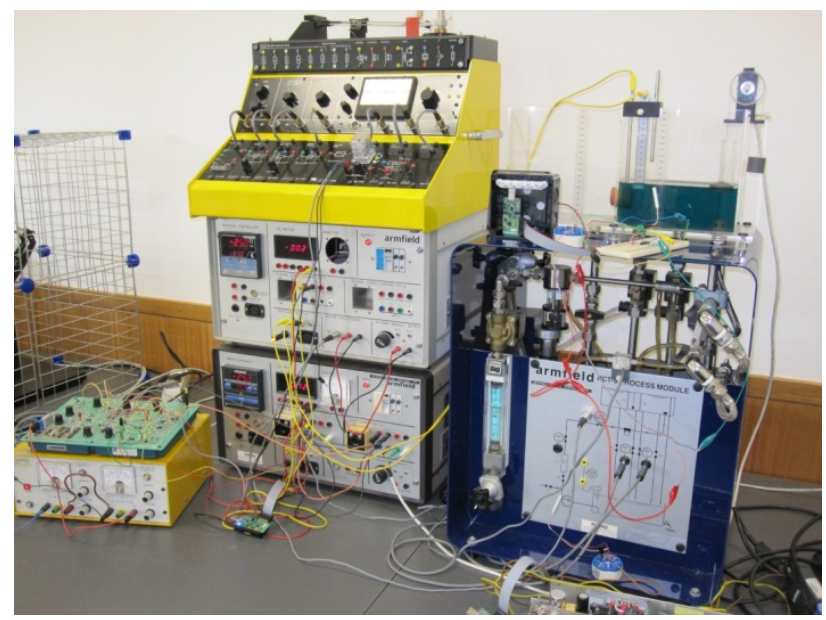

Figure 7. The two-tank process (РСТ9) used in Experiment 2. 
The three-tank system (model DTS200 made by Amira) is a benchmark system that could be used in this experiment, considering a WSAN to acquire the system outputs and send the system inputs. With this setup users can implement and test a remote controller (on the platform's client side, Fig. 8) that receives the tanks' levels and sends the control actions to the two pumps (actuators) through the network.

\section{Experiment 4: Wireless Networked Control System}

The goal of this experiment is to incorporate a wireless communication network between the controller and a MIMO system to build up a networked environment.

In this experiment (Fig. 9) a supervisor and a Distributed Control System (DCS) of a MIMO system over a WSAN is considered. Several aspects concerning the supervision of distributed systems, networked control systems and WSAN communication protocols could be studied.

\section{CONCLUSION}

This paper aims to point out that wireless sensor and actuator networks can be a relevant, low cost and very effective infrastructure to allow interaction with real laboratory systems for online experimentation purposes. Teaching and online learning activities, especially in engineering courses could benefit from using WSAN to implement and carry out online experiments for different subjects. WSAN constitute a flexible and accurate interface to remotely acquire data from laboratory systems and send back data under the form of command or control

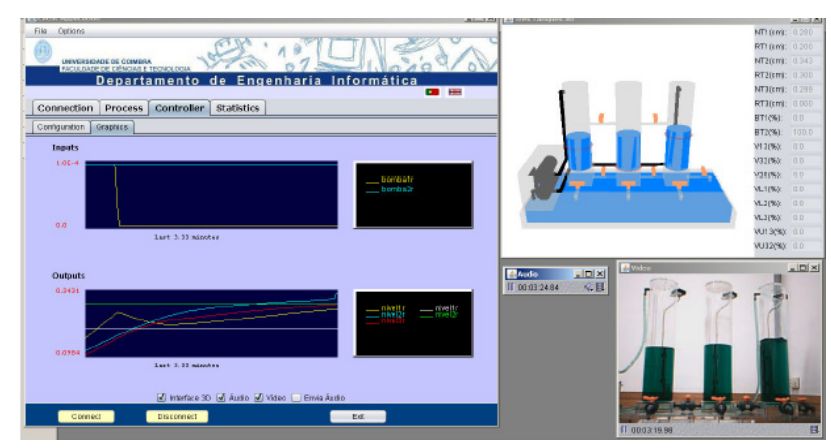

Figure 8. Interface of the remote lab using the three-tank system (DCS200) in Experiment 3.

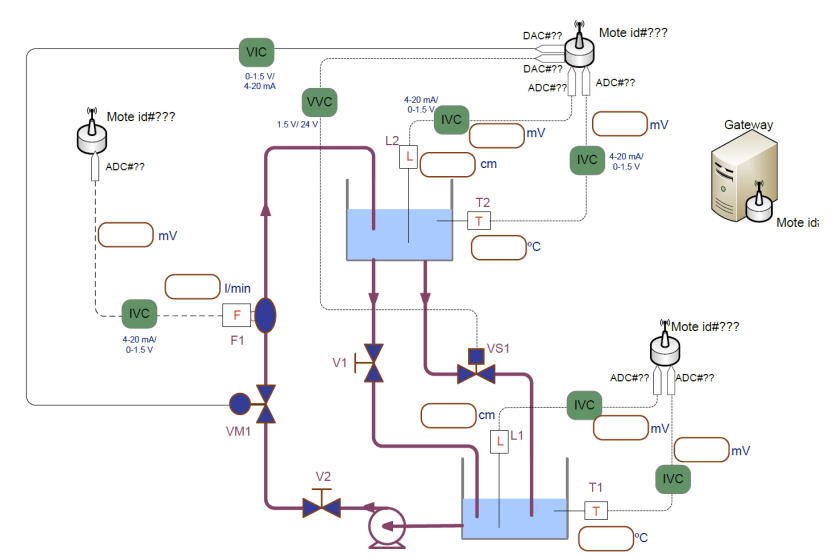

Figure 9. The diagram of the Distributed Control System based on a WSAN used in Experiment 4. actions. They can be found in a large range of application areas and they can be used to simplify the interaction with real labs for learning purposes.

The referred flock.uc.pt web platform aims to contribute to a new paradigm of learning and training using online experimentation, enabling to develop a system rich in educational contents that is practical, flexible and easy to access, breaking down spatial and temporal barriers of the traditional approaches.

Students and trainees may accomplish their own experiments from her/his laptop, just requiring the availability of an internet connection to have access to different kinds of virtual or real plants and experiments in a e- or b-learning context and for enriching the class room activities.

Some examples of remote experiments using WSAN were presented to illustrate the feasibility and flexibility of this approach.

\section{ACKNOWLEDGMENT}

The authors wish to acknowledge the support provided by the Centre for Informatics and Systems of the University of Coimbra (CISUC) for the development of this project.

\section{REFERENCES}

[1] D. R. Garrison and N. D. Vaughan, Blended Learning in Higher Education: Framework, Principles, and Guidelines, John Wiley \& Sons, Inc., 2008.

[2] W. S. Swail, "Higher education and the new demographics: Questions for policy. Change Magazine, 34(4), pp. 15-23, 2002.

[3] F. Newman, L. Couturier and J. Scurry, The future of higher education: Rhetoric, Reality, and the Risks of the Market, JosseyBass, 2004.

[4] N. S. Edward, "The role of laboratory work in engineering education: student and staff perceptions”, International Journal of Electrical Engineering Education, vol. 39, no. 1, pp. 11-19, 2002.

[5] M. E. Auer, A. Pester and D.G Zutin, "Open Source Portal for Online Laboratories”, Proceedings of the 4th International Conference on Remote Engineering and Virtual Instrumentation REV 2007. Kassel University Press, 2007.

[6] Wilson, S., O. Liber, M. Johnson, P. Beauvoir, P. Sharples and C. Milligan. "Personal Learning Environments: Challenging the dominant design of educational systems", Journal of e-Learning and Knowledge Society, vol.3 (2), 2007.

[7] I. F. Akyildiz, W. Su, Y. Sankarasubramaniam and E. Cayirci, "Wireless Sensor Networks: a survey", Computer Networks, April 2002, pp.393-422. http://dx.doi.org/10.1016/S1389-1286(01)003 $\underline{02-4}$

[8] A. Cardoso, M. Vieira and P. Gil, "A Remote and Virtual Lab with Experiments for Secondary Education, Engineering and Lifelong Learning Courses", iJOE Special Issue: exp.at'11 Conference, Vol. 8, pp. 49-54, 2012.

[9] A. Cardoso, M.T. Restivo, P. Cioga, M. Delgado, J.N. Monsanto, J. Bicker, E. Nunes and P. Gil, "Demonstration of Online Educational Modules with Online Experiments", Proceedings of REV2012 International Conference, July, 2012.

[10] M. Ohlin, D. Henriksson, and A. Cervin, "TrueTime 1.5 Reference Manual”, Department of Automatic Control, Lund University, Sweden, 2007. Available at: http://www.control.lth.se/truetime (accessed in December 2012).

[11] A. Cardoso, S. Santos, A. Santos and P. Gil, "Simulation Platform for Wireless Sensor Networks based on the TrueTime Toolbox", Proceedings of IECON2009 International Conference, November, 2009. 


\section{AUTHORS}

A. Cardoso is with the CISUC - Centre for Informatics and Systems of the University of Coimbra, Department of Informatics Engineering, University of Coimbra, Polo II, 3030-290 Coimbra, Portugal (e-mail: alberto@dei.uc.pt).

P. Gil is with the CISUC - Centre for Informatics and Systems of the University of Coimbra and with the Departamento de Engenharia Electrotécnica, Faculdade de Ciências e Tecnologia da Universidade Nova de Lisboa, Campus de Caparica, Lisboa, Portugal (e-mail: psg@fct.unl.pt).

This article is an extended and modified version of a paper presented at the IGIP2012 conference, held 26 - 28 September 2012, in Villach, Austria. Received 17 December 2012. Published as resubmitted by the authors 28 January 2013.. 